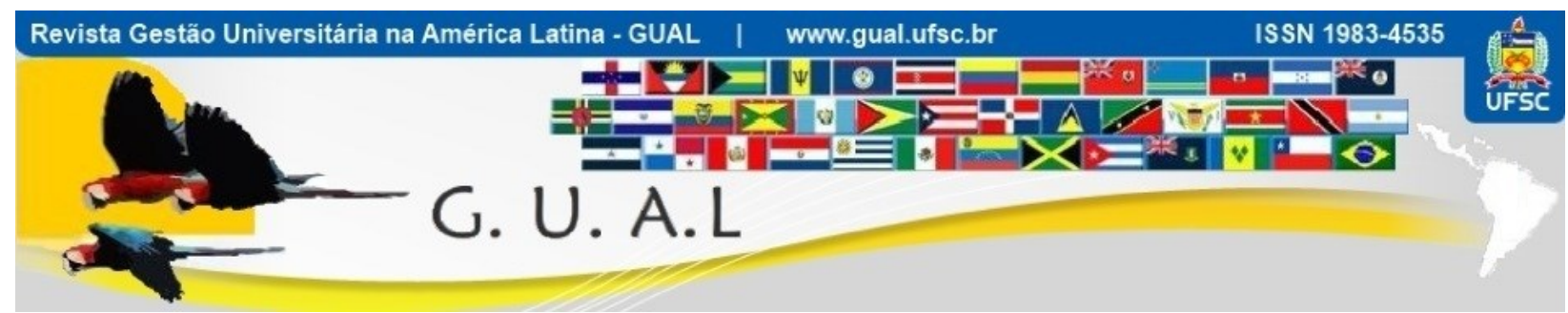

DOI: http://dx.doi.org/10.5007/1983-4535.2018v11n1p229

\title{
RANKINGS UNIVERSITÁRIOS MUNDIAIS: ANÁLISE DA CONGRUÊNCIA ENTRE OBJETIVOS E INDICADORES
}

\section{RANKINGS UNIVERSITY WORLD: CONGRUENCE ANALYSIS OF GOALS AND INDICATORS}

Sandra Mara Iesbik Valmorbida, Doutoranda

Universidade Federal de Santa Catarina - UFSC Universidade Tecnológica Federal do Paraná - UTFPR smiesbik@gmail.com

Thuine Lopes Cardoso, Mestra Universidade Federal de Santa Catarina - UFSC thuinecardoso@hotmail.com

Sandra Rolim Ensslin, Doutora Universidade Federal de Santa Catarina - UFSC sensslin@gmail.com

Recebido em 05/maio/2016

Aprovado em 28/novembro/2017

Sistema de Avaliação: Double Blind Review

Esta obra está sob uma Licença Creative Commons Atribuição-Uso. 


\title{
RESUMO
}

O caráter internacional do ensino superior leva estudantes, universidades e governos a ter um interesse crescente em conhecer a posição das universidades em comparação com outras no contexto mundial. Os rankings são atraentes à medida que fornecem estas informações. Entretanto, cada um dos rankings desenvolvido faz uso de indicadores e métricas de mensuração definidos segundo o propósito para o qual ele foi criado. Este trabalho tem por objetivo investigar, com base nas publicações científicas, se há congruência entre os indicadores utilizados pelos rankings universitários com os objetivos para os quais o ranking foi desenvolvido. Para isso, foi realizada busca de artigos científicos nas bases Web of Science e na SCOPUS, que resultou em 25 artigos que foram então analisados. Trata-se de uma pesquisa bibliográfica, de natureza qualitativa, com base em dados secundários. Assim, primeiramente identificaram-se os rankings apresentado pelos trabalhos, sendo que apenas foram analisados os que apareceram em quatro ou mais trabalhos: ARWU; THES; HEEACT; CWTS; Webometrics; SCImago; QS; America's Best College. Com base na análise, foi possível constatar que dos rankings analisados, dos quais apresentaram seus indicadores, todos apresentam alinhamento entre indicadores e objetivos, mesmo que alguns casos sejam de forma genérica e superficial.

Palavras-chave: Rankings Universitários. Análise da Literatura. Indicadores de Desempenho.

\begin{abstract}
The international character of higher education takes students, universities and governments have a growing interest in knowing the position of universities compared to others in the global context. The rankings are attractive as they provide this information. However, each of the rankings developed makes use of indicators and metrics of measurement defined according to the purpose for which it was created. This work aims to investigate, based on scientific publications, if there is congruence between the indicators used by university rankings with the objectives for which the ranking was developed. For this search was conducted for scientific articles in web basis of Science and SCOPUS, which resulted in 25 articles were then examined. This is a bibliographic research, qualitative in nature, based on secondary data. So, first we identified the rankings presented by the work, and only analyzed those who appeared in four or more jobs: ARWU; THES; HEEACT; CWTS; Webometrics; SCImago; QS; America's Best College. Based on the analysis, it was found that the analyzed rankings, which presented their indicators, all present alignment of indicators and targets, although some cases are generic and superficial way.
\end{abstract}

Keywords: University Rankings. Analysis of the Literature. Performance Indicators. 


\section{INTRODUÇÃO}

A globalização do ensino superior permitiu que as universidades pudessem competir internacionalmente tanto por recursos econômicos como por recursos humanos (ROCHA et al., 2012; AGUILLO et al., 2010). De acordo com Bornmann, Mutz e Daniel (2013) existe uma longa história de competição entre as universidades, onde elas competem por alunos, professores e por questões financeiras. Assim, devido ao aumento da concorrência e à internacionalização do ensino superior, rankings universitários são criados para medir o desempenho das instituições, permitindo comparações e classificações entre as universidades (BORNMANN et al., 2013).

A classificação das universidades tornou-se inevitável, uma vez que as mesmas estão cada vez mais globais e tem diversos stakeholders internos e externos. Os alunos, por exemplo, precisam dos rankings para escolher onde estudar, os cientistas para saber onde trabalhar, o governo para saber onde investir e os dirigentes da universidade para analisar o seu desempenho (CHARON; WAUTERS, 2007).

Dessa forma, os rankings universitários servem para ajudar na decisão de potenciais alunos e colaboradores de universidade ou instituição de pesquisa em relação à escolha da instituição mais bem avaliada na área geográfica de interesse; e fornecem aos governos ferramentas para avaliar a eficácia do ensino superior (BORNMANN et al., 2013; BENITO; ROMERA, 2011).

Porém, os rankings acadêmicos são considerados um assunto um tanto controverso, uma vez que existem vários sistemas classificatórios para universidades e instituições de pesquisa e não há consenso sobre o que é qualidade de uma instituição de ensino superior (BENITO; ROMERA, 2011), o que pode afetar a credibilidade dos rankings, haja vista que acaba gerando resultados divergentes.

Esses resultados divergentes devem-se ao processo de desenvolvimento dos rankings, pois são desenvolvidos por diversas instituições ao redor do mundo, além de ter necessidades informacionais que indicam objetivos diferentes (BUELA-CASAL et al., 2007). Assim, é possível constatar semelhanças e diferenças em termos de objetivos, estrutura, indicadores e atribuição de pesos (grau de importância dos indicadores). Isso influencia o resultado apresentado pelo(s) ranking(s) (HUANG, 2012). Por isso, os rankings têm recebido críticas (BENITO; ROMERA, 2011), podendo-se citar que comparam contextos e universidades que não são comparáveis (BORNMANN; ANEGÕN; MUTZ, 2013); utilizam indicadores que são 
difíceis de reproduzir e, às vezes, requerem o uso de opiniões (FLORIAN, 2007); são fortemente baseadas em dados bibliométricos (AGUILLO et al., 2010); não são representativos, de forma suficiente, do que pretendem avaliar, e os resultados são tendenciosos em relação a certos países (AGUILLO et al., 2010). Isso se dá porque cada ranking é constituído para uma finalidade e não pode, então, ser utilizado por todos os interessados, caso os interesses não estejam alinhados.

Nesse sentido, Benito e Romera (2011) afirmam que desenvolver uma metodologia de classificação universal capaz de fornecer dados de desempenho comparável, confiável e internacional sobre as instituições de ensino superior é uma atividade complexa. Bornmann et al. (2013) complementam que se deve reconhecer a diversidade das instituições e considerar os diferentes objetivos das insituições no momento da avaliação e classificação. $\mathrm{O}$ mesmo deve ser levado em consideração quando da observação do resultado de um ranking: o que ele mede e qual o objetivo para o qual foi construído.

Com isso, surge o seguinte questionamento: Os rankings universitários atendem ao objetivo para o qual são construídos? A fim de buscar resposta para essa questão, este trabalho tem por objetivo investigar se há congruência entre os indicadores utilizados pelos rankings universitários com o objetivo para o qual o ranking foi desenvolvido.

Assim, justifica-se a realização deste trabalho quanto à importância, à originalidade e à viabilidade (CASTRO, 1977). A presente pesquisa é importante porque analisa a congruência entre os objetivos e os indicadores utilizados pelos principais rankings universitários encontrados na literatura. É original, por não ter sido encontrado, na literatura consultada, outros trabalhos que se propusessem a verificar seus objetivos e atendimento. Percebe-se viável pela facilidade de acesso aos dados, visto que os artigos que serão objetos de análise são buscados no portal de periódicos da CAPES e nos sites que divulgam os rankings na internet.

Este trabalho limita-se a analisar apenas os rankings citados por quatro ou mais artigos encontrados no fragmento da literatura consultado. As informações omissas nos artigos foram buscadas no site que divulga o ranking na internet.

Além desta seção introdutória, este artigo apresenta o referencial teórico na seção dois; a metodologia, na seção três; os resultados são apresentados na seção quatro; as considerações finais, na seção cinco; e, por fim, as referências base para este trabalho são apresentadas. 


\section{REFERENCIAL TEÓRICO}

\subsection{RANKINGS UNIVERSITÁRIOS}

Com a massificação das universidades, praticamente em todos os continentes, as iniciativas para obter uma análise da qualidade das universidades têm aumentado rapidamente nos últimos anos. O caráter internacional do ensino superior leva estudantes, universidades e governos a ter um interesse crescente em conhecer a posição das universidades em comparação com outras similares (BUELA-CASAL et al., 2007).

A educação superior tornou-se tão internacional que já não é suficiente, para as universidades, conhecer sua posição em comparação com universidades do seu próprio país. Com a competitividade global é necessário comparar com as universidades de diversos países (BUELA-CASAL et al., 2007). Assim, o sucesso dos rankings se dá devido à globalização do ensino superior, em que uma universidade pode competir internacionalmente por recursos econômicos e humanos (AGUILLO et al., 2010). De acordo com Bornmann, et al. (2013), há uma longa história de competição entre as universidades, elas competem por alunos, professores e por questões financeiras.

Como as universidades e o ensino superior estão cada vez mais globais e possuem diversos stakeholders internos e externos, a aplicação de uma classificação das atividades da universidade tornou-se inevitável (CHARON; WAUTERS, 2007). Nesse contexto, é quase impossível falar sobre a classificação e a avaliação das instituições de ensino superior e, consequentemente, fazer uma comparação, sem se referir aos rankings acadêmicos (BERNARDINO; MARQUES, 2010).

As listas de classificação visam informar as posições com base em indicadores de qualidade do desempenho acadêmico. Essas classificações informam os usuários, ou seja, os tomadores de decisão e formuladores de políticas, os alunos e seus pais, os financiadores de pesquisas, etc., sobre a qualidade de universidades e suas performances, subsidiando, assim, a qualidades de suas decisões (LINDBLAD, 2008).

Os alunos usam dessas classificações para escolher onde estudar, os cientistas para saber onde trabalhar, os governos para saber onde investir e os dirigentes da universidade para saber como está o seu desempenho a fim de melhorar sua performance (CHARON; WAUTERS, 2007). De acordo com Huang (2012), os rankings desempenham um papel importante não somente no recrutamento de estudantes, mas também na dotação orçamentária governamental para o ensino superior. 
Quando se trata do ensino superior, os rankings acadêmicos são um assunto um tanto controverso. Muitos aspectos críticos são apontados para os rankings de universidades, e uma das principais críticas é que as universidades são diferentes (BERNARDINO; MARQUES, 2010). Neste contexto, para Bernardino e Marques (2010) duas instituições diferentes, com objetivos e missões distintas, não podem ser comparadas. De acordo com Turner (2013), uma universidade que vê sua missão como sendo servir uma região que se estende além das fronteiras nacionais irá desempenhar um papel diferente de uma universidade que vê a sua missão como sendo a de servir uma região dentro de um único país. Assim, com suas missões e objetivos diferentes, as universidades não são passíveis de comparação, distorcendo os resultados dos rankings universitários. Porém, mesmo com todas as críticas, a tendência de uso das informações geradas pelos rankings, parece consolidar-se e cada vez mais diferentes usuários os utilizam para obter informações sobre o desempenho das instituições de ensino superior (BERNARDINO; MARQUES, 2010).

Nesse contexto, o objetivo principal dos rankings é dar informações aos diversos interessados a fim de ajudá-los a fazer escolhas referentes ao ensino superior e às universidades. Porém, internamente (para as universidades), existem outros objetivos como o de funcionar como uma estratégica de marketing e estimular a busca pela qualidade da instituição de ensino (BUELA-CASAL et al., 2007).

Os resultados dos rankings não permitem apenas a avaliação da qualidade do ensino superior, mas também afetam a competitividade no mercado educacional. A fim de melhorar a competitividade e para entrar em rankings internacionais, as prioridades de diversas universidades incluem o desenvolvimento das seguintes medidas: melhoria do potencial intelectual da universidade, atraindo os principais cientistas e professores, suporte para jovens cientistas, promoção internacional da pesquisa e inovação e, finalmente, incentivos aos professores e pesquisadores visando melhorar o desempenho da instituição (RODIONOV; RUDSKAIA; KUSHNEVA, 2014).

Normalmente, as instituições de ensino superior não gostam de ser classificadas e comparadas às outras, porém, no atual contexto, a comparação e os rankings são inevitáveis. Contudo, apesar das diversas críticas que os rankings acadêmicos enfrentam, o principal objetivo de diversas universidades é estar no topo dos rankings acadêmicos, devido à sua popularidade, o que possibilita uma boa reputação e uma publicidade gratuita (BERNARDINO; MARQUES, 2010). 
Buela-Casal et al. (2007) afirmam que as origens dos rankings universitários são tradicionalmente associadas aos meios de comunicação de massa e às empresas de publicidade. Portanto, são frequentemente utilizados como ferramenta de marketing pelas universidades, visando comprovar sua excelência de ensino e pesquisa (JEREMIC; MILENKOVIC, 2014).

\subsection{AVALIAÇÃO DE DESEMPENHO - INDICADORES}

Os indicadores de desempenho estão sendo utilizados nos mais diferentes contextos, desde a gestão de instituições de saúde até a gestão das universidades (LINDBLAD, 2008). No âmbito universitário, de acordo com Marginson (2014), os gestores estão sendo pressionados a adotar políticas destinadas a melhorar a classificação nos rankings e, para isso, fazem uso dos indicadores de desempenho para apoiar sua gestão.

$\mathrm{Na}$ maioria dos rankings acadêmicos, os indicadores são escolhidos devido à possibilidade de serem medidos pelo fácil acesso aos dados e às informações, em vez de serem escolhido devido à realmente serem significativos para avaliação das instituições (BERNARDINO; MARQUES, 2010). Os sistemas de classificação das universidades podem variar amplamente, dependendo do tipo de indicadores selecionados e da definição particular de qualidade acadêmica adotada pelos seus autores (BUELA-CASAL et al., 2007). Assim, a seleção dos indicadores, bem como a percepção dos autores dos rankings afetam seus resultados, atribuindo-lhes um caráter ad hoc (subjetivo). Cumpre observar que essa característica de subjetividade em nada desqualifica o caráter científico do ranking, apenas direciona a concepção filosófica, as estratégias de investigação e o método da pesquisa a serem utilizadas.

De acordo com Jeremic e Milenkovic (2014), os rankings são atraentes à medida que fornecem informações sobre determinada universidade no contexto mundial, porém a simplicidade de uso pode ser altamente enganosa, à medida que desconsidera o alinhamento entre o propósito para o qual foi construído e os métodos de pesquisa utilizados com a resposta encontrada e seu uso.

Os pesos atribuídos a esses indicadores nos rankings universitários também são controversos. Alguns especialistas argumentam que a escolha de pesos muitas vezes carece de uma base teórica. Outra preocupação diz respeito à alteração do valor dos pesos e/ou indicadores, sem informação aos usuários: se os pesos ou os indicadores são alterados a cada 
edição, isso significa que o resultado, ou a "qualidade" da instituição também será alterado. Assim, torna-se muito difícil interpretar e direcionar as mudanças no desempenho da instituição rumo ao aperfeiçoamento sem o conhecimento das alterações ocorridas (BERNARDINO; MARQUES, 2010).

Bernardino e Marques (2010) concluem que, para serem úteis, os indicadores devem ter base científica, confiabilidade e reduzir a subjetividade (aqui sendo referenciada a evidenciação/explicitação para entendimento/conhecimento de todos). A coleta dos dados deve ser coerente, disponível e transparente. Os pesos atribuídos devem aparecer com base nas conclusões de extensa análise da contribuição de cada indicador para o desempenho da instituição.

\section{METODOLOGIA}

\subsection{ENQUADRAMENTO METODOLÓGICO}

Os procedimentos técnicos utilizados nesta pesquisa são: a pesquisa bibliográfica e a pesquisa-ação (RICHARDSON, 1999). Entende-se que é bibliográfica por envolver a análise de artigos anteriormente publicados; e é pesquisa-ação pela interação entre os pesquisadores e os resultados durante toda a operacionalização do instrumento ProKnow-C, para a seleção do Portfólio Bibliográfico (PB) que resulta de várias delimitações e inferências que os pesquisadores fazem durante a sequência do processo estruturado. A interação também ocorre no mapeamento das características, estabelecidas pelos autores que permitem a análise de congruência do PB.

No que se refere à coleta de dados, o objetivo deste estudo faz uso de dados secundários, uma vez que os artigos científicos estão disponíveis, on-line, nas bases de dados do portal de periódicos da CAPES (RICHARDSON, 1999).

Quanto à abordagem do problema, classifica-se como qualitativa (Richardson, 1999), por responder, por meio da análise da congruência, que tem por base o princípio de utilidade (BENITO; ROMERA, 2011) dos "rankings universitários".

\subsection{PROCEDIMENTOS PARA COLETA E ANÁLISE DOS DADOS}

O objetivo deste trabalho é investigar se há congruência entre os indicadores utilizados pelos rankings universitários com o objetivo para o qual o ranking foi criado. Para isso, 
inicialmente faz-se necessária à formação do Portfólio Bibliográfico (PB) sobre o tema "Rankings Universitários”, para, em seguida, proceder à sua análise.

A formação do $\mathrm{PB}$ se dá com artigos relacionados ao fragmento da literatura relativo ao tema, e a análise sistêmica, aqui representada pela análise da congruência, desses artigos será realizada mediante o desenvolvimento das etapas sequenciais do instrumento Knowledge Development Process-Constructivist (ProKnow-C) (DUTRA et al., 2015; ENSSLIN et al., 2014a; ENSSLIN et al., 2014b; VALMORBIDA et al., 2014; ENSSLIN; ENSSLIN; PINTO, 2013; LACERDA; ENSSLIN; ENSSLIN, 2012; TASCA et al., 2010).

Para a formação do PB, fez-se a busca nas bases Web of Science e Scopus, com as palavras "ranking" AND "university". Delimitou-se que a busca seria por título, resumo e palavras-chave, apenas artigos, sem limite temporal. Dessa busca, resultaram 4.771 artigos. A partir desse resultado, foram realizadas filtragens, primeiro pela leitura dos títulos para verificar o alinhamento ao tema desta pesquisa, nessa fase eliminaram-se 4.596 artigos. Em seguida, observou-se o reconhecimento científico, em que se identificaram 123 artigos. Verificou-se a disponibilidade desses artigos gratuitamente na internet, dos quais 52 não foram encontrados. Dos 71 artigos restantes, foi feita a leitura dos resumos e, posteriormente, a leitura completa dos artigos. Após essa leitura foram selecionados 32 artigos como representativos do fragmento da literatura relativo ao tema rankings universitários.

Com o portfólio selecionado, foram identificados os rankings analisados. Delimitou-se que, para a análise sistêmica, apenas seriam objeto de análise os rankings apresentados por quatro ou mais trabalhos. Assim, foram eliminados dessa análise sete trabalhos, resultando em 25 trabalhos, que serão objetos deste estudo.

$\mathrm{Na}$ sequência, os autores analisaram os artigos do PB, buscando identificar: i) o ranking apresentado; ii) o objetivo para o qual foi criado; iii) quem participa da sua construção; iv) os usuários a que se destinam; v) os indicadores utilizados para a classificação; vi) conformidade entre indicadores e objetivos; e, vii) se os indicadores são passíveis de ser gerenciados pelas instituições avaliadas. Cabe salientar que, quando divergentes ou ausentes as informações de interesse deste trabalho, elas foram buscadas na página oficial do ranking na internet. Cumpre salientar que algumas informações não foram encontradas em nenhum dos dois locais. Salienta-se ainda que, quando se analisam os indicadores, será observado, o objetivo que o indicador representa bem como as métricas adotadas para esta mensuração, por exemplo: indicador citação (genérico, pode ser mensurado 
de diversas formas), deve explicitar o que busca mensurar, por exemplo: número de artigos publicados em periódicos indexados no Scopus (específico e transparente).

A análise da congruência será realizada tendo por base o princípio de utilidade, conforme apresentado por Benito e Romera (2011). Segundo o autor, um sistema de ranking para ser útil deve indicar claramente os objetivos para o qual foi construído para, assim, construir indicadores alinhados com o objetivo do ranking e os interesses do público a que se destina ou que será influenciado por ele.

\section{APRESENTAÇÃO E DISCUSSÃO DOS RESULTADOS}

A partir da seleção de artigos que tratavam sobre o tema "rankings universitários" foi realizada a leitura e análise do conteúdo desse PB, visando identificar: i) o ranking apresentado; ii) o objetivo para o qual foi criado; iii) quem participa da construção do ranking; iv) os usuários a que se destinam; v) os indicadores utilizados para a classificação; vi) a conformidade entre indicadores, objetivos e usuários; e, vii) indicadores passíveis de serem gerenciados pelas instituições avaliadas.

Inicialmente, foram identificados os rankings apontados pelos trabalhos analisados, conforme demonstrados na Figura 1. Cabe salientar que apenas foram analisados os que foram objeto de estudos de quatro ou mais trabalhos. 
Figura 1 Principais Rankings Universitários Mundiais e Artigos que o citam

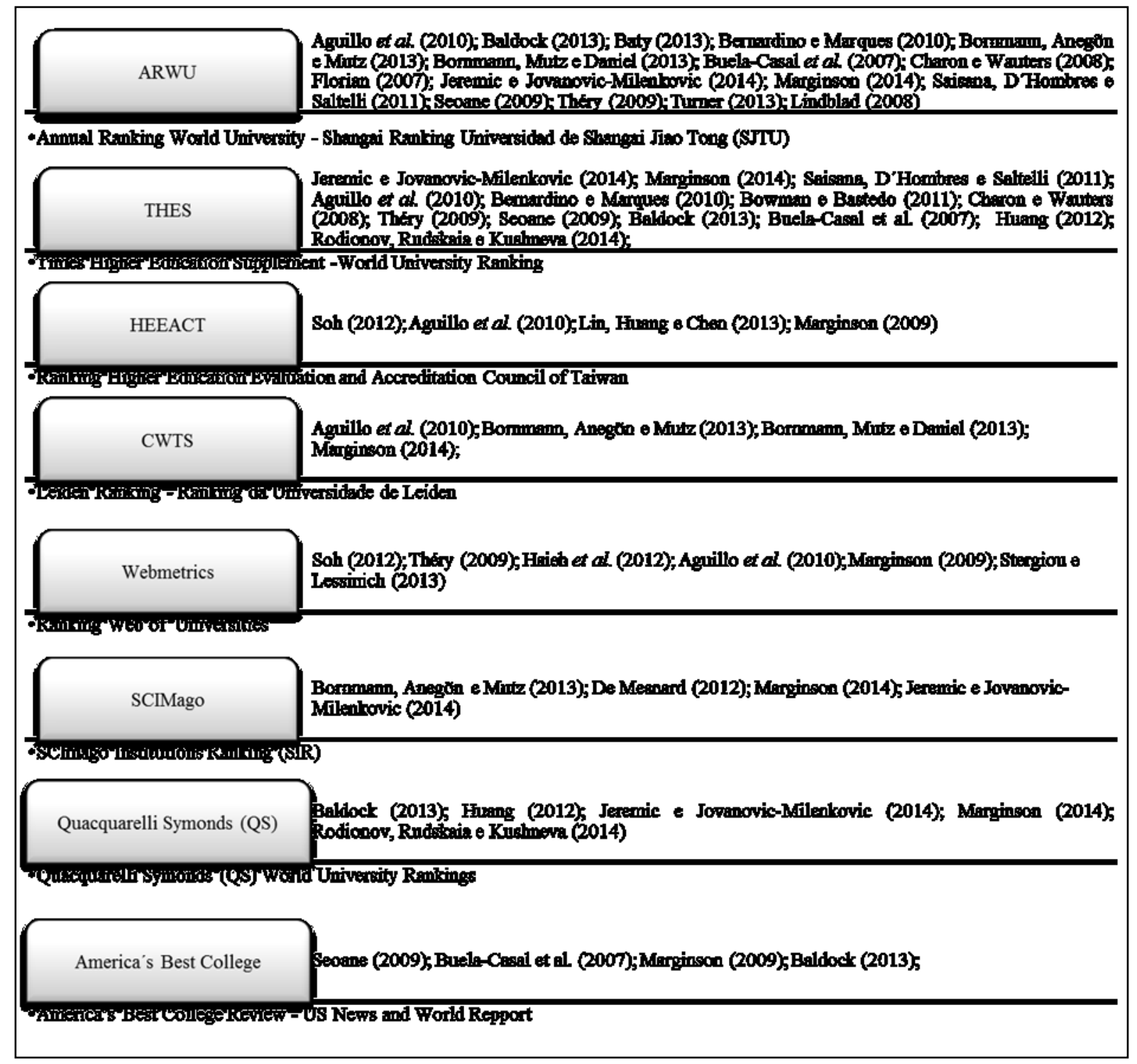

Fonte: Dados da Pesquisa (2015).

Percebe-se, pelo exposto na Figura 1, que o ARWU é a classificação anual mais utilizada pelos trabalhos e também a mais conhecida e utilizada pelas universidades e instituições de pesquisa do mundo. Com base nesses trabalhos e na análise do website dos rankings é que foram analisados os objetivos, os usuários e os indicadores observados que serão relatados a seguir.

O Academic Ranking of World Universities (ARWU) é publicado anualmente, desde 2003, pela Universidade de Jiao Tong de Shangai, da China, para conhecer a posição das universidades chinesas comparadas com universidades mundialmente reconhecidas, também visando identificar as instituições de prestígio internacional para estabelecer parcerias. Assim, 
tem por usuário as instituições de ensino, que visem buscar parceiros de pesquisa e o governo chinês para saber a posição de suas universidades. Mais de 1.200 universidades são analisadas pelo ARWU cada ano, e as 500 melhores são publicadas. Concentra-se no desempenho acadêmico e de pesquisa das universidades e para isso usa seis indicadores: o número de exalunos vencedores do Prêmio Nobel e da Medalha Field (10\%) e membros do corpo docente que obtiveram tais prêmios $(20 \%)$; o número de pesquisadores altamente citados na Web of Science - Thomson Reuters (20\%); o número de artigos publicados nas revistas Nature e Science (20\%); o número de artigos indexados no Science Citation Index - Expanded e Social Sciences Citation Index (20\%); e o atendimento docente por aluno (10\%).

Um indicador que chama a atenção é o número de vencedores do Prêmio Nobel que pertencem ou pertenceram à instituição. Embora seja uma importante propaganda para a instituição, é discutível que essa premiação impacte no desempenho acadêmico ou na pesquisa. Já os indicadores de citações e publicações de pesquisadores e artigos indexados são medidas adequadas à qualidade da pesquisa realizada, no entanto, pouco remete ao desempenho no ensino, que, nesse ranking, é avaliado apenas pelo número de estudantes atendidos pelo professor. Neste sentido, uma vez que o objetivo do ranking é estabelecer parcerias, tendo como um dos usuários as instituições de ensino que buscam parceiros de pesquisa, os indicadores utilizados adéquam-se ao objetivo à medida que apenas o indicador: atendimento docente por aluno não é voltado para a pesquisa.

Em relação à possibilidade de ser gerenciado, observou-se que a maioria dos indicadores são de difícil gerenciamento como as citações, publicações em revistas e em periódicos indexados e o Prêmio Nobel, onde poucas ações ou ações a longo prazo, contribuem para que a universidade consiga melhorar seu desempenho neste aspecto. Apenas o indicador que mede o desempenho docente é passível de ser plenamente gerenciado.

O Times Higher Education Supplement - World University Ranking (THES), publicado anualmente pela Thomson Reuters, tem por finalidade avaliar o desempenho das melhores universidades nas áreas de ensino, pesquisa, extensão e internacionalização. Busca atender a vários usuários: estudantes, acadêmicos, líderes universitários, mercado e governo. Para isso, empregam 13 indicadores de desempenho agrupados em cinco áreas: Ensino: ambiente de aprendizagem (30\%); Pesquisa: volume, renda e reputação (30\%); Citações: influência de pesquisa (30\%); Geração de renda para indústria: inovação $(2,5 \%)$; Visibilidade 
internacional: atração de funcionários e alunos; e Visibilidade da pesquisa (7,5\%). São excluídas as instituições com menos de 200 citações por ano.

Como tanto o objetivo quanto os indicadores dos rankings são abrangentes e estão relacionados, considerou que existe uma conformidade entre os mesmos. Porém, critica-se a efetiva utilidade e a informação gerada pelo mesmo, uma vez que o ranking fornece um resultado genérico, utilizando apenas 13 indicadores para atender a diversos objetivos. Quanto às métricas utilizadas, como as mesmas não são explicitadas, apenas as áreas, não é possível analisar precisamente o gerenciamento dos indicadores.

O Ranking Higher Education Evaluation and Accreditation Council of Taiwan (HEEACT), publicado anualmente, desde 2006, pelo Higher Education Evaluation and Accreditation Council of Taiwan, realiza avaliações institucionais e de programas em universidades e faculdades taiwanesas para conduzir projetos de garantia de qualidade e melhoria de pesquisa e desenvolvimento do ensino superior. Busca ainda aumentar a visibilidade e facilitar a internacionalização das instituições de Taiwan. Não especifica os destinatários da informação, porém, baseado nesse objetivo, entende-se que tem por usuários as instituições de ensino que visem buscar internacionalização e o governo de Taiwan para saber a posição de suas universidades. Utiliza como indicadores: número de publicações e citações de acordo com as bases de dados de citação Web of Science da Thomson Reuters (Science Citation Index, Science Citation Index, Social Essential Science Indicators), dando especial atenção às publicações recentes.

Os indicadores utilizados por esse ranking estão relacionados especificamente à pesquisa realizada pela instituição do que a avaliação institucional e a qualidade das instituições, o que corrobora a pesquisa de Robinson (2013). A visibilidade internacional dessas instituições fica comprometida, pois estará limitada à pesquisa, haja vista não existirem indicadores relacionados à qualidade do ensino para atração de alunos e professores estrangeiros. Assim, os indicadores estão em conformidade apenas com um dos objetivos dos rankings.

Cabe salientar que esses indicadores são de difícil gerenciamento pela instituição avaliada, onde poucas ações ou ações a longo prazo, contribuem para melhorar seu desempenho.

O Leiden Ranking (CWTS), publicado anualmente, desde 2007, pelo Centre for Science and Technology Studies, da Universidade de Leiden (Holanda), apresenta como 
finalidade avaliar a qualidade da pesquisa feita pelas instituições. Mede o impacto científico de 750 grandes universidades em todo o mundo. Embora não especificados claramente, os autores entendem que podem ser usuários desse ranking os pesquisadores para buscar locais para trabalhar, os investidores na área de pesquisa e ainda as instituições de pesquisa, para conhecerem sua posição em relação às demais.

Usa um conjunto de indicadores bibliométricos: volume de trabalhos científicos; volume de citações desses artigos; citações por artigo, o número de artigos no topo de citação de sua área; a proporção dos artigos da universidade na base de dados Web of Science da Thomson Reuters. Mais de 1.000 trabalhos são necessários para que uma universidade seja classificada entre as 750 universidades com maior produção científica.

Os indicadores de citações e publicações de pesquisadores e artigos indexados são medidas adequadas à qualidade da pesquisa realizada, estando de acordo com os objetivos do ranking.

Quanto ao gerenciamento pelas instituições, entende-se que como tratam basicamente de indicadores bibliométricos, são indicadores de difícil gerenciamento e há possibilidade de ações de longo prazo por parte da instituição para que possam melhorar a posição no ranking.

O Ranking Web ou Webometrics publicado anualmente, desde 2004, pelo Laboratório Cybermetrics (Conselho Nacional de Pesquisa Espanhol) para promover visibilidade acadêmica na web, apoiando as iniciativas de acesso livre para aumentar significativamente a transferência de conhecimento científico e cultural gerado pelas universidades para toda a sociedade e fornecer informações úteis em tempo real para os gestores de sites universitários, os quais são identificados como usuários dessa informação.

Os indicadores utilizados por esse ranking são construídos da seguinte forma: visibilidade (50\%): a qualidade dos conteúdos é avaliada por um "referendo virtual", contando todos os links externos que o site da universidade recebe de terceiros; esses links reconhecem o prestígio institucional, o desempenho acadêmico, o valor da informação, bem como a utilidade dos serviços introduzido nas páginas de acordo com os critérios de milhões de editores web de todo o mundo; atividade (50\%): presença (1/3): número total de páginas hospedadas no domínio web (incluindo todos os subdomínios e diretórios) da universidade como indexados pelo maior motor de busca comercial (Google); abertura (1/3): número de arquivos (pdf, doc, docx, ppt) publicados em sites dedicados de acordo com o mecanismo de 
pesquisa acadêmica Google Scholar; e excelência (1/3): número total de publicações mais citados em seus respectivos campos científicos (10\%).

Esse ranking está voltado ao atendimento de necessidades informacionais dos gestores universitários em relação à acessibilidade e visibilidade de sua instituição. Dessa forma, analisando os indicadores apresentados percebe-se que os mesmos estão em conformidade com o objetivo considerando fatores externos e internos da visibilidade acadêmica. No entanto, a ponderação de pesos para essa visibilidade é imposta pelos organizadores da classificação. Assim, falham no atendimento ao fornecimento de informações úteis aos gestores, pois cada gestor pode julgar diferentemente o que é uma informação útil.

Acredita-se que os indicadores de visibilidade referem-se a medidas externas que dificilmente conseguiriam ser gerenciadas pela instituição, e o contrário ocorre com a maioria dos indicadores de atividade.

O SCImago Institutions Ranking (SIR), publicado anualmente, desde 2009, pela base de dados Scopus, seu objetivo é avaliar a qualidade de organizações de pesquisa, e não apenas universidades. Embora não especificados pelos organizadores, e tendo em conta seu objetivo, os autores entendem que poderiam ser usuários desse ranking os pesquisadores para buscar locais para trabalhar, os investidores na área de pesquisa e ainda as instituições de pesquisa para conhecerem sua posição em relação às demais. Para avaliação da qualidade, utiliza único indicador: o número total de documentos publicados em revistas acadêmicas indexadas na Base Scopus. Utilizar um único indicador não é problema, porém deve-se reconhecer a capacidade limitada desse indicador em responder por uma questão tão ampla como a qualidade das organizações de pesquisa. Da forma como está desenhado, esse ranking limitase à informação da Base Scopus (mantenedora do ranking) em identificar a quantidade de publicações de cada instituição nessa base de dados. Porém, mesmo que de forma limitada, o indicador utilizado está em conformidade com o objetivo do ranking. Cumpre salientar que,

Como se trata de indicador de indexação pode ser trabalhado internamente para que os pesquisadores busquem publicação em periódicos indexados à Base Scopus, assim a instituição pode gerenciar, a longo prazo, o resultado a fim de melhorar sua posição no ranking.

O Quacquarelli Symonds (QS) World University Rankings, publicado anualmente, desde 2004, tem por objetivo ajudar os estudantes a fazer comparações entre as opções de estudo internacionais. Considera mais de 3.000 instituições e compara as 800 melhores 
universidades de quatro grandes áreas de interesse para os futuros alunos: ensino, pesquisa, empregabilidade e internacionalização.

Essas quatro áreas-chave são avaliadas utilizando seis indicadores, aos quais é dada uma ponderação percentual diferente: (i) Reputação acadêmica (40\%): acadêmicos de todo o mundo são convidados a identificar o local onde eles acreditam que o melhor trabalho está ocorrendo atualmente dentro do seu campo de especialização; (ii) Reputação do empregador (10\%): empregadores identificam as universidades que consideram produzir os melhores graduados; (iii) Relação de Docente/número alunos matriculados (20\%): número de docentes empregados em relação ao número de alunos matriculados; (iv) Citações por faculdade (20\%): número de citações que os artigos de pesquisadores da universidade têm, coletadas da Base Scopus, base de dados mundial de resumos de pesquisa e citações. O total de citação é avaliado em relação ao número de membros do corpo docente acadêmico da universidade; (v) Atração de professores estrangeiros (5\%): número de professores estrangeiros em relação ao total de docentes; e (vi) Proporção de estudantes estrangeiros (5\%): número de alunos estrangeiros em relação ao total de discentes.

Esse ranking deixa claro que tem por usuário os alunos que pretendam escolher onde estudar, tendo como objetivo ajudá-los a fazer comparações entre suas opções e os indicadores utilizados estão em conformidade com esse objetivo. Com esse foco, coleta vários indicadores que contemplam áreas-base das instituições, porém não atende ao seu princípio de utilidade, conforme apresentado por Benito e Romera (2011), à medida que cada aluno pode atribuir pesos diferentes a cada área avaliada com base no seu ponto de vista, o que resultaria numa classificação diferente a cada aluno, haja vista a divulgação que o ranking leva em conta as atribuições de pesos fornecidas pelos seus desenvolvedores.

Quanto à possibilidade de ser gerenciado, observou-se que alguns indicadores são de difícil gerenciamento, necessitando de ações de longo prazo, tais como citações, publicações em revistas e em periódicos indexados, atração de professores e alunos estrangeiros. Apenas os indicadores referentes ao número de professores estrangeiros em relação ao total de docentes; proporção de estudantes estrangeiros número de alunos estrangeiros em relação ao total de discentes; relação de docente/número alunos matriculados; e o número de docentes empregados em relação ao número de alunos matriculados são de fácil gerenciamento.

O America's Best College Review, publicado anualmente, desde 1983, pelo US News and World Repport, é citado pelos trabalhos como o primeiro ranking de universidades. Tem 
por finalidade medir a qualidade acadêmica das universidades públicas e privadas da América do Norte, para orientar os alunos a escolher a melhor instituição para estudar.

Para essa mensuração, usa como critérios: avaliação por pares - reputação $(25 \%)$; retenção $(20 \%)$ para as universidades nacionais e faculdades de artes liberais $(25 \%)$; recursos do corpo docente (20\%); seleção de alunos (15\%); recursos financeiros (10\%); desempenho na graduação (5\%); e recursos por alunos $(5 \%)$.

O ranking não fornece as métricas da mensuração, apenas a área de avaliação e como as mesmas não são explicitadas não são possíveis analisar precisamente o gerenciamento dos indicadores e a conformidade com o objetivo.

Esse ranking foca questões relacionadas ao ensino e à administração da universidade, não privilegiando questões relacionadas à pesquisa, que estes pesquisadores entendem importante para avaliar a qualidade acadêmica. Outro aspecto observado que fragiliza esse ranking é a avaliação por pares que pergunta a opinião de várias pessoas envolvidas com a academia sobre qual universidade considera melhor para que um aluno escolha, além de remeter à falta de objetividade na resposta.

Ainda, cabe salientar que, como os usuários da informação são alunos que estão procurando local para cursarem sua graduação, logo cada aluno pode atribuir pesos diferenciados para os aspectos considerados pelo ranking.

Com base nas análises individuais realizadas, pode-se objetivamente comparar os vários rankings encontrados, conforme apresentado no Quadro 1.

Quadro 1 Comparativo dos rankings mais citados na literatura consultada

\begin{tabular}{|c|c|c|c|c|c|}
\hline Ranking & $\begin{array}{l}\text { Apresenta } \\
\text { Objetivo? }\end{array}$ & $\begin{array}{l}\text { Apresenta os } \\
\text { indicadores } \\
\text { usados? }\end{array}$ & $\begin{array}{l}\text { Conformidade entre } \\
\text { indicadores } \\
\text { objetivos? }\end{array}$ & $\begin{array}{l}\text { Gerenciável pela } \\
\text { instituição } \\
\text { avaliada? }\end{array}$ & $\begin{array}{l}\text { Identifica } \\
\text { o(s) } \\
\text { usuário(s)? }\end{array}$ \\
\hline ARWU & Sim & Sim & Sim & Parcialmente & Sim \\
\hline THES & Sim & Não & -* & $-*$ & Sim \\
\hline HEEACT & Sim & Sim & Sim & Parcialmente & Não \\
\hline CWTS & Sim & Sim & Sim & Parcialmente & Não \\
\hline Webometrics & Sim & Sim & Sim & Parcialmente & Sim \\
\hline SCImago & Sim & Sim & Sim & Parcialmente & Não \\
\hline QS & Sim & Sim & Sim & Parcialmente & Sim \\
\hline America's & Sim & Não & $-*$ & $-*$ & Sim \\
\hline
\end{tabular}

Fonte: Dados da Pesquisa (2015).

* Não foi possível julgar em função da não especificação das métricas utilizadas. 
Com base no Quadro 1, é possível visualizar que dos rankings analisados, que apresentaram seus indicadores, todos apresentam alinhamento entre indicadores e objetivos, mesmo que alguns casos sejam de forma genérica e superficial. Outro ponto a ser destacado é que os indicadores podem ser parcialmente gerenciados pelas instituições, uma vez que a maioria dos indicadores, para ser gerenciado, necessitam de ações a longo prazo e sofrem influências externas. Assim, torna-se difícil para as instituições ter o controle da posição no ranking, comprometendo o sistema como forma de avaliação de desempenho.

Realizou-se também análise dos rankings à luz das críticas encontradas no referencial teórico. Os trabalhos de Aguillo et al. (2010); Amsler (2013); Bornmann et al. (2013); e Huang (2012) argumentam que os rankings são fortemente baseados em dados bibliométricos, o que foi constatado com o ARWU; THES; HEEACT; CWTS e SCImago.

Há críticas de que eles não são transparentes (ou seja, não deixam claro quais as métricas que estão utilizando, e de onde tiram os dados) (BENITO; ROMERA, 2011; FLORIAN, 2007). Mesmo quando consultados, os sites não apresentam abertamente os indicadores utilizados, como é o caso de THES; QS e America's; de que não são representativos, sendo usados para uma pluralidade de cenários (AGUILLO et al., 2010; BENITO; ROMERA, 2011; JEREMIC; MILENKOVIC, 2014) como ocorrem com THES; HEEACT; CWTS; CWTS; Webometrics; QS e America's;

Outros trabalhos criticam a arbitrariedade na atribuição de pesos dos indicadores (BENITO; ROMERA, 2011; BUELA-CASAL et al.; 2007; FLORIAN, 2007); e de que os rankings são irreproduzíveis (FLORIAN, 2007), como é o caso do ARWU; THES; HEEACT; CWTS; QS; America's.

Benito e Romera (2011); Boulton (2011); Buela-Casal et al. (2007) apontam que os rankings comparam instituições que não podem ser comparáveis, porque têm missões e contextos diferentes. Isso foi constatado no que ocorre com ARWU; HEEACT; SCImago e America's.

\section{CONCLUSÃO}

Este trabalho teve por objetivo investigar se há congruência entre os indicadores utilizados pelos rankings universitários com o objetivo para o qual o ranking foi desenvolvido. Para isso, foi realizada uma busca de artigos científicos nas bases Web of 
Science e na SCOPUS, conforme demonstrado na seção 3.2. Resultou em 25 artigos que foram então analisados.

Assim, primeiro identificaram-se os rankings apresentado pelos trabalhos, sendo que apenas foram analisados os rankings que apareceram em quatro ou mais trabalhos. Foram analisados: (i) Academic Ranking of World Universities (ARWU); (ii) Times Higher Education Supplement-World University Ranking (THES); (iii) Ranking Higher Education Evaluation and Accreditation Council of Taiwan (HEEACT); (iv) Leiden Ranking - Centre for Science and Technology Studies (CWTS); (v) Ranking Web ou Webometrics; (vi) SCImago Institutions Ranking (SIR); (vii) Quacquarelli Symonds (QS) World University Rankings; (viii) America's Best College Review - US News and World Repport.

Desses, oito diferentes rankings foram objeto de análise de conteúdo: (i) o objetivo para o qual foi criado; (ii) quem participa da construção do ranking; (iii) os usuários a que se destinam; (iv) os indicadores utilizados para a classificação; (v) conformidade entre indicadores e objetivos; e, (vi) os indicadores são passíveis de ser gerenciados pelas instituições avaliadas. A congruência foi julgada tendo por base o princípio de utilidade, conforme apresentado por Benito e Romera (2011). Segundo os autores, um sistema de ranking para ser útil deve indicar claramente os objetivos para o qual foi construído e identificar com precisão o público abordado (objetivo a que se destina) para, assim, construir indicadores alinhados com o objetivo do ranking e aos interesses do público a que se destina ou que será influenciado por ele. A análise foi feita individualmente, e posterior análise global, conforme apresentado no Quadro 1.

Com base nessa análise, foi possível constatar dos rankings analisados que apresentaram seus indicadores e métricas utilizadas, todos apresentam alinhamento entre indicadores e objetivos, mesmo que alguns casos sejam de forma genérica e superficial. Outro ponto a ser destacado é que os indicadores podem ser parcialmente gerenciados pelas instituições, uma vez que a maioria dos indicadores, para ser gerenciado, necessita de ações, a longo prazo, e sofrem por influências externas.

Dessa forma, é possível responder à pergunta-base deste trabalho: Os rankings universitários atendem ao objetivo para o qual são construídos? Concluiu-se que os referidos rankings, atendem ao objetivo para o qual foram construindo, também foi possível verificar que há congruência entre os objetivos e indicadores utilizados. Porém cumpre salientar que, embora sejam utilizados amplamente por vários usuários, os rankings falham em relação ao 
princípio global da utilidade, pois não levam em conta as necessidades informacionais dos usuários da informação embora a maioria deles cumpra alguma característica identificada como necessária.

Aponta-se que os resultados desta pesquisa não podem ser generalizados para os demais rankings universitários, à medida que cabe análise das particularidades de cada sistema. Sugere-se para futuras pesquisas a ampliação do número de rankings avaliados.

\section{REFERÊNCIAS}

AGUILLO, I. F.; BAR-ILAN, J.; LEVENE, M.; ORTEGA, J. L. Comparing university rankings. Scientometrics, v. 85, n. 1, p. 243-256, 2010.

ARWU. Academic Ranking of World Universities. Disponível em: $<$ http://www.arwu.org/>. Acesso em 20 de março de 2015.

BALDOCK, C. University rankings and medical physics. Australasian Physical and Engineering Sciences in Medicine, v. 36, n. 4, p. 375-378, 2013.

BATY, P. The times higher education world university rankings, 2004-2012. Ethics in Science and Environmental Politics, v. 13, n. 2, p. 125-130, 2013.

BENITO, M.; ROMERA, R. Improving quality assessment of composite indicators in university rankings: A case study of French and German universities of excellence. Scientometrics, v. 89, n. 1, p. 153-176, 2011.

BERNARDINO, P.; MARQUES, R. C. Academic rankings: An approach to rank Portuguese universities. Ensaio, v. 18, n. 66, p. 29-48, 2010.

BORNMANN, L.; ANEGÕN, F. M.; MUTZ, R. Do universities or research institutions with a specific subject profile have an advantage or a disadvantage in institutional rankings?: A Latent Class Analysis with Data from the SCImago Ranking. Journal of the American Society for Information Science and Technology, v. 64, n. 11, p. 2310-2316, 2013.

BORNMANN, L.; MUTZ, R.; DANIEL, H. D. Multilevel-statistical reformulation of citation-based university rankings: The Leiden ranking 2011/2012. Journal of the American Society for Information Science and Technology, v. 64, n. 8, p. 1649-1658, Aug 2013. 
BOWMAN, N. A.; BASTEDO, M. N. Anchoring effects in world university rankings: Exploring biases in reputation scores. Higher Education, v. 61, n. 4, p. 431-444, 2011.

BUELA-CASAL，G.; GUTIÉRREZ-MARTÍNEZ，O; BERMÚDEZ-SÁNCHEZ，M. P.; VADILLO-MUÑOZ, O. Comparative study of international academic rankings of universities. Scientometrics, v. 71, n. 3, p. 349-365, 2007.

CASTRO, C. M. A prática da pesquisa. São Paulo: McGraw-Hill do Brasil, 1977.

CHARON, A.; WAUTERS, J. P. University ranking: a new tool for the evaluation of higher education in Europe. Nephrology Dialysis Transplantation, v. 23, n. 1, p. 62-64, Jan. 2008.

DE MESNARD, L. On some flaws of university rankings: The example of the SCImago report. Journal of Socio-Economics, v. 41, n. 5, p. 495-499, 2012.

DUTRA, A.; RIPOLL-FELIU, V.M.; FILLOL, A. G.; ENSSLIN, S. R.; ENSSLIN, L. The construction of knowledge from the scientific literature about the theme seaport performance evaluation, International Journal of Productivity and Performance Management, v. 64, n. 2, p. 243 - 269, 2015. http://dx.doi.org/10.1108/IJPPM-01-2014-0015.

ENSSLIN, S. R.; RIPOLl-FEliU, V. M.; ENSSLIN, L.; DUTRA, A. Performance Evaluation to Support the University Management Activity. La Pensée (Paris), v. 76, p. 2$17,2014 a$.

ENSSLIN, S. R.; ENSSLIN, L.; IMLAU, J. M.; CHAVES, L. C. Processo de mapeamento das publicações científicas de um tema: portfólio bibliográfico e análise bibliométrica sobre avaliação de desempenho de cooperativas de produção agropecuária. Revista de Economia e Sociologia Rural (Impresso), v. 52, p. 587-608, 2014 b.

FLORIAN, R. V. Irreproducibility of the results of the Shanghai academic ranking of world universities. Scientometrics, v. 72, n. 1, p. 25-32, 2007.

GIL, A. C. Métodos e técnicas de pesquisa social. 5. ed. São Paulo: Atlas, 1999.

HEEACT. Ranking Higher Education Evaluation and Accreditation Council of Taiwan. Disponível em: $<\underline{\text { http://www.heeact.edu.tw/mp.asp? } \mathrm{mp}=4}>$. Acesso em 20 de março de 2015. 
HSIEH, S. M.; LO, S. A.; HSU, C. C.; LIN, W. C. Novel cloud service for improving world universities ranking. In: Proceedings of the 9th International Conference on Information Technology, ITNG 2012, 2012. Anais Eletrônicos., 2012. Disponível em: < http://www.scopus.com/inward/record.url?eid=2-s2.0-

84863931111\&partnerID=40\&md5=7ff87c300e76ea10dca6b9d5fca60326 >.

HUANG, M. H. Opening the black box of QS world university rankings. Research Evaluation, v. 21, n. 1, p. 71-78, 2012.

JEREMIC, V.; MILENKOVIC, M. J. Evaluation of Asian university rankings: Position and perspective of leading Indian higher education institutions. Current Science, v. 106, n. 12, p. 1647-1653, 2014.

LACERDA, R. T. O.; ENSSLIN, L.; ENSSLIN, S. R. Uma Análise Bibliométrica da literatura sobre Estratégia e Avaliação de Desempenho. Gestão \& Produção, v. 19, n.1, p. 59-78, 2012.

LEIDEN. Leiden Ranking Centre for Science and Technology Studies. Disponível em: $<$ http://www.leidenranking.com/>. Acesso em 20 de março de 2015.

LIN, C. S.; HUANG, M. H.; CHEN, D. Z. The influences of counting methods on university rankings based on paper count and citation count. Journal of Infometrics, v. 7, n. 3, p. 611-621, 2013.

LINDBLAD, S. Navigating in the field of University positioning: On international ranking lists, quality indicators and higher education governing. European Educational Research Journal, v. 7, n. 4, p. 438-450, 2008.

MARGINSON, S. Open source knowledge and university rankings. Thesis Eleven, v. 96, n. 1, p. 9-39, 2009.

. University rankings and social science. European Journal of Education, v. 49, n. 1, p. 45-59, 2014.

QS. Quacquarelli Symonds (QS) World University Rankings. $<$ http://www.topuniversities.com/qs-world-university-rankings $>$. Acesso em 20 de março de 2015. 
ROCHA, A. C.; MATOS, O. A.; VENÂNCIO, D.; DUTRA, A. Gestão estratégica em instituições de ensino superior: mapeamento das publicações científicas no período de 1997 a 2012. Revista Gestão Universitária na América Latina - GUAL, v. 7, p. 106-127, 2014.

RODIONOV, D. G.; RUDSKAIA, I. A.; KUSHNEVA, O. A. The importance of the university world rankings in the context of globalization. Life Science Journal, v. 11, n. 10 SPEC. ISSUE, p. 442-446, 2014.

SAISANA, M.; D'HOMBRES, B.; SALTELLI, A. Rickety numbers: Volatility of university rankings and policy implications. Research Policy, v. 40, n. 1, p. 165-177, 2011.

SCIMAGO Institutions Ranking. Disponível em: <www.scimagoir.com/ > Acesso em 20 de março de 2015.

SEOANE, A. Y. ¿Cómo se evalúan las universidades de clase mundial? Revista de la educación superior, v. 38, n. 150, p. 113-120, 2009.

SILVA, R. V.; ENSSLIN, S. R.; RIPOLL-FELIU, V.; SOLER, C. C.. E-government and Public Accounting Information: Bibliometric and Systemic Analysis. International Research Journal of Finance and Economics, Issue 122, 2014.

$\mathrm{SOH}, \mathrm{K}$. World university rankings: What is in for top ten East Asian universities? New Horizons in Education, v. 60, n. 2, 2012.

STERGIOU, K. I.; LESSENICH, S. On impact factors and university rankings: From birth to boycott. Ethics in Science and Environmental Politics, v. 13, n. 2, p. 101-111, 2013.

TASCA, J. E.; ENSSLIN, L.; ENSSLIN, S. R.; ALVES, M. B. M. An approach for selecting a theoretical framework for the evaluation of training programs. Journal of European Industrial Training, v. 34, n. 7, p. 631-655, 2010.

THÉRY, H. The academic ranking of world universities: Shanghai and the others. Mappemonde, v. 96, n. 4, 2009.

THES. Times Higher Education Supplement -World University Ranking. Disponível em: $<$ http://www.timeshighereducation.co.uk/>. Acesso em 20 de março de 2015. 
TURNER, D. A. World class universities and international rankings. Ethics in Science and Environmental Politics, v. 13, n. 2, p. 167-176, 2013.

US. America's Best College Review. Disponível em: <http://www.usnews.com/>. Acesso em 20 de março de 2015.

VALMORBIDA, S. M. I.; ENSSLIN, S. R.; ENSSLIN, L.; RIPOLL-FELIU, V. M.. Avaliação de Desempenho para Auxílio na Gestão de Universidades Públicas: Análise da Literatura para Identificação de Oportunidades de Pesquisas. Contabilidade, Gestão e Governança, v. 17, n. 3, p. 4-28, 2014.

WEBOMETRICS. Disponível em: <http://www.webometrics.info/>. Acesso em 20 de março de 2015.

THES. Times Higher Education Supplement -World University Ranking. Disponível em: $<$ http://www.timeshighereducation.co.uk/>. Acesso em 20 de março de 2015. 